\title{
Références
}

\section{Les cartes conceptuelles dans les formations en santé}

\author{
Claire MARCHAND*, Jean-François d'IVERNOS*
}

\begin{abstract}
Messagesclés
- Lesthéories cognitives del'apprentissage apportent un éclairagesur la façon dont l'apprenant construit ses connaissances En effet, l'organi sation des connaissances constituerait une étape essentielle au développement de l'expertise. • La carte conceptuelle est un des moyens proposés pour représenter la structure cognitive, et donc l'organisation des connaissances, d'un individu. • La carte conceptuelle présente différentsintérêts: elle favorisel'apprenti ssage en le rendant plus s gni ifiant pour l'apprenant, elle constitue pour les enseignants un moyen de réflexion pour la planification de leur enseignement, elle permet d'évaluer la façon dont un apprenant intègre et organise ses connaissances • Les modal ités d'élaboration ed d'analyse d'une carte conceptuelle sont variables, qu'elle soit élaborée individuellement ou en groupe. $\bullet \mathrm{L}$ a carte conceptuelle est utilisée en éducation, dans différentes disciplines et dansles formations médicaleset paramédicales Elle consitue actuelle ment un thème de recherche et d'application dansle domaine de l'éducation thérapeutique des patients.
\end{abstract}

\section{Mots clés}

Carte conceptuelle ; organisation des connaissances ; apprentissage signifiant ; évaluation de l'apprentissage ; planification del'enseignement ; éducation thérapeutique du patient.

\section{Key Messages}

- The theories of cognitive psychology enlighten the process by which a learner built his knowledge The organization of knowledge constitutesan essential step of the development of expertise ${ }^{\circ}$ C oncept mapping is one of the poss bl ewaysto dis play the cognitive scaffolding and consequently the knowledge organization. ${ }^{\circ}$ Concept mapping offers many advantages: it promotes meaningful learning among students, it helpstheteacher in structuring his courseand curriculum, it allowsto better evaluate how thelearner integrates and manages his knowledge. ${ }^{-T}$ They are varioustechniques to built and analyse concept maps (individually or in group). • C oncept mapping is widespread in education, in variousdisciplines and in the medical and nursing curriculum. It is actually experienced in the fied of patient therapeutic education.

\section{Keys Words}

Concept mapping; knowledge organization; meaningful learning; learning assessment; instructional planning; patient therapeutic education.

Pédagogie M édicale $2004 ; 5$ : 230-240

\footnotetext{
*Laboratoire de pédagogie de la Santé - UPRES EA 3412 - Université Paris 13 - URR SMBH Léonard de Vinci 74 rue Marcel Cachin - 93017 Bobigny Cedex - France

Correspondance: Caire MARCHAND - Laboratoire de Pédagogie de la Santé - UPRES EA 3412 - Université Paris 13 UFR SMBH Léonard de Vinci - 74 rue Marcel Cachin - 93017 Bobigny Cedex - France - Tél. : (33) 148388898 Fax : (33) 148387619 - mailto:c.marchand@smbh.univ-paris13.fr
} 


\section{Cadre théorique des cartes conceptuelles}

L'utilisation descartes conceptuellessinspire des principes de la cartographie'. D éveloppéebien avant lelangage écrit, la cartographie a constitué un signe du développement humain majeur. Elle représente un instrument de communication humainequi, commetout moyen de communication, met en jeu un émetteur et un récepteur. Celui qui élabore la carte, mais aussi celui qui va la lire, réalisent un ensembled'opérations mentales pour en extrairelesens (détecter, reconnaître, différencier, estimer, etc.). Le système de cartographie et son interprétation requièrent une transformation cognitive complèteà la fois intellectuelleet visuelle. II s'agit donc d'un exercice de construction, de savoir ou de construction de sens. Les théories cognitivistes de l'apprentissage incitent à sintéresser à la façon dont l'étudiant construit ses connaissances, à comprendre comment il les organise dans sa structure mentale. Pour de nombreux auteurs', 3, 4, 5, 6 |'organisation des connaissances détermine en partie la façon dont elles vont pouvoir être utilisées pour résoudre un problème et prendre des décisions. Elle constituerait une étape essentielle pour le développement de l'expertise dans un domaine particulier et serait prédictive de compétences mentales complexes.

Les moyens utilisés pour représenter les connaissances d'un individu et leur organisation sinspirent des principes de fonctionnement de la mémoire à long terme (enregistrement, codification, stockage). On trouve, par exemple, des représentations sous la forme de schémas, de scripts, etc., mais aussi de réseaux conceptuels?.

Une carte conceptuelle est une représentation écrite, hié rarchisée d'un ensemble de concepts et de relations de sens qui les relient. L'élaboration d'une carte conceptuelle per- met d'extérioriser la pensée d'un individu à travers l'agencement des conceptsqu'il met en jeu. Elleobligel'individu à penser dans différentes directions. Ellerequiert de sa part une compréhension profonde du thème traité et l'aide à penser 8 .

G énéralement, la méthode d'élaboration d'une carte conceptuelle comporte plusieurs étapes (Tableau 1) et nécessite un réd apprentissage tant de la part des enseignants que des apprenants ${ }^{7}$.9. A partir de cette méthode de base, différentes variantes sont proposées pour réaliser des cartes conceptuelles : élaboration libre, à partir d'un ensemble de concepts et/ou de liens prédéterminés, carte préélaborée à compléter, etc., ${ }^{90}$.

\section{Utilisation des cartes conceptuelles dans le domaine de la formation médicale et para médicale}

A titre d'exemple, la figure 1 reprend sous la forme d'une carte conceptuelle, les principaux aspects développés dans le paragraphe suivant.

\section{Une aide à l'apprentissage}

Initialement, les cartes conceptuelles ont été proposées pour représenter la structure cognitive d'un individu et favoriser chez les apprenants un apprentissage signifiant (qui a du sens, meaningful learning) plutôt qu'un apprentissage par cœur (routinier, rote learning)7, 11 En effet, l'élaboration par un apprenant d'une carte conceptuelle lui permet de clarifier ses connaissances, de mettre en relief

\section{Tableau 1 : Étapes d'élaboration d'une carte conceptuelle'}

1. A partir d'un thème, d'un concept, d'une compétence, etc. L'étidiant détermine les principaux concepts qui semblent associés. On obtient une liste de concepts qui peuvent représenter des objets, des événements, des symboles, etc.

2. A partir de cette liste de concepts, organiser les connai ssances en classant les concepts du plus général au plus spécifique, du plus abstrait au plus concret. On définit ainsi des concepts dit de «superordination » et des concepts de « subordination » (Ausubel, 1968).

3. Trier ensuite les concepts en fonction de leur niveau d'abstraction et de leurs relations afin de les agencer ou de les situer sur la carte.

4. Relier les différents concepts entre eux par une flèche et une expression verbale indiquant le sens de la relation. II peut s'agir d'un verbe, d'un nom, d'une conjonction de coordination ou d'un terme de liaison. 


\section{Références}

les idées essentielles, de schématiser et de hiérarchiser ses savoirs. Le plus souvent, au moment de l'élaboration de la carte conceptuelle, l'apprenant doute de ses connaissances, ce qui suscite chez lui une motivation à apprendre. Plusieurs étude ${ }^{12,13}$ ont montré que les cartes conceptuelles augmentai ent la compréhension et améliorai ent les capacités de résolution de problème. En biologie par exemple, les cartes conceptuelles, comparées aux stratégies d'enseignement et d'apprentissage classiquement utilisées, favorisent l'assimilation de nombreux concepts et de relations complexes ${ }^{12}$.

Par ailleurs, les cartes conceptuelles améliorent la mémorisation, la mise en réseau des connaissances économisant la mémoire de travail ${ }^{14}$. En effet, le regroupement des informations en réseau rend plus accessibles les connaissances quand on veut les utiliser. Par exemple, les cartes conceptuelles ont été empl oyées pour améliorer l'intégration et la mémorisation de concepts en pharmacologi $e^{15}$, pour aider des apprenants à mettre en lien les données théoriques et pratiques telles que les éléments d'un processus de soins chez les infirmiers ${ }^{16,17}$. Certains auteurs décrivent l'intérêt des cartes conceptuelles pour aider les étudiants à planifier des soins infirmiers. Dans ce cas, il est proposé aux étudiants infirmiers d'élaborer des cartes conceptuelles à partir d'un cas clinique complexe (clinical concept mapping) ${ }^{18,19}$. Les informations importantes sont identifiées, puis hiérarchisées et mises en lien explicitement par les étudiants. Pour ces auteurs, cette technique développe chez les apprenants leur capacité métacognitive, donne du sens à leur apprentissage et les entraîneà développer unevision plus gl obaledes patients dont ils s'occupent.

Initialement élaborées de façon individuelle, les cartes conceptuelles peuvent aussi être construites en groupe. Comme cela a été démontré dans d'autres domaine ${ }^{20}$, les interactions suscitées par l'échange de connaissances sont sources de nombreux apprentissages par les conflits cognitifs qu'elles provoquent au sein du groupe. D es études ont montré que les étudiants qui construisaient des cartes conceptuelles en groupe acquéraient de meilleures connaissances que les étudiants travaillant individuellement ${ }^{21,22}$. La carte conceptuelle devient un outil de négociation de sens, non seulement entre les étudiants mais aussi en interaction avec l'enseignant. Certains auteurs se sont particulièrement intéressés aux mécanismes se produisant au cours de l'élaboration d'une carte conceptuelle en groupe, tant au niveau des apprenants qu'à celui de I'enseignant ${ }^{23,24}$. La carte conceptuelle sert de support à
I'enseignant pour questionner les apprenants sur leurs connaissances. L'explicitation des connaissances par les étudiants eux-mêmes suscite chez eux une réflexivité sur leur propre savoir, développe ainsi leur capacité méta cognitive et améliore l'intégration des connai ssances ${ }^{25}$. D ans le domaine médical et paramédical, des expériences d'utilisation des cartes conceptuelles élaborées individuellement ou en groupe ont été menées dans le cadre d'un apprentissage par problèmes. $D$ ans certaines facultés de médecine (U niversités de $G$ enève ${ }^{26}$ et de Sherbrook ${ }^{27}$, par exemple), mais aussi dans le cadre de la formation des infirmiers ${ }^{19}$, l'élaboration d'une carte conceptuelle constitue parfois une étape de la démarche d'apprentissage par problèmes (APP). Elle permet aux étudiants d'organiser en groupe ou individuellement leurs connaissances sur le cas présenté et de générer des hypothèses explicatives.

Elle sert aussi de référence pour donner un fedback explicite écrit aux étudiants qui ont réal isé une carte conceptuelle individuellement à l'issue de leur temps de travail personnel ${ }^{27}$. Enfin, la carte conceptuelle peut aussi constituer une activité d'autoformation. Au cours d'une forma tion pédagogique auprès de professionnels de santé (euxmêmes formateurs) ${ }^{28}$, certains d'entre eux ont volontairement participé à une expérience d'autoformation en élaborant individuellement, à distance des sessions de formation et à différents moments de leur cursus, une carte conceptuelle à partir du concept de métacognition. Conjointement à l'élaboration des cartes conceptuelles, les participants devaient répondre à des questions d'ordre réflexif témoignant de leur processus d'apprentissage de ce concept. Au-delà d'un véritable travail sur la clarification et l'organisation des connaissances autour du concept de métacognition, cette expérience a permis de développer chez les professionnels concernés leurs propres capacités métacognitives.

\section{Une aide à la planification}

Les cartes conceptuelles peuvent être égal ement utilisées par les enseignants pour construire leur programme d'enseignement?. En faisant apparaitre les concepts principaux à enseigner, la carte conceptuelle incitel'enseignant à sélectionner les informations essentielles, à les structurer et par conséquent à organiser la manière dont elles doivent être transmises. C e type d'organisation préalable, présentée régulièrement aux étudiants, favoriserait un apprentissage structurééc,30

Aux Etats-Unis, les cartes conceptuelles sont utilisées pour 
la planification des curricula basés sur l'apprentissage par problèmes ou encore pour l'élaboration des cas problèmes ${ }^{11}$. D e même, les cartes conceptuelles constituent des outils permettant d'améliorer la collaboration interdisciplinaire en facilitant la visual isation des articulations possibles entre différentes disciplines ${ }^{31,32}$. A ssociées, par exemple, à un apprentissage par problèmes, les cartes conceptuelles se sont révééés très utiles pour travailler les aspects transdisciplinaires et multidisciplinaires de l'inté gration d'un module spécifique sur la santé de la femme dans les études médicales ${ }^{3}$. Enfin, les cartes conceptuelles constituent d'excellents supports pour aider les enseignants à sélectionner des objectifs d'apprentissage pertinents à partir de problèmes à traiter ou de compétences à développer ${ }^{34}$.

\section{Un outil d'évaluation}

Les cartes conceptuelles permettent d'éval uer les connaissances des apprenants. Elles peuvent être utilisées à différents moments en fonction des intentions évaluatives. $D$ ans le cadre d'une évaluation diagnostique, elles permettent d'orienter la définition d'objectifs d'apprentissage pertinents en favorisant la visualisation des connaissances préal ables des apprenants ainsi que leurs incompréhensions, leurs lacunes, leurs erreurs. Au cours de la formation, la carte conceptuelle constitue un outil d'évaluation formative car elle permet d'ajuster le programme aux besoins des apprenants et de guider ceux-ci dans leurs apprentissages ${ }^{35}$. C'est principalement dans cette perspective que les concepteurs de la carte conceptuelle ont suggéré leur utilisation?. En effet, la carte conceptuelle représente un excellent outil de révélation des conceptions erronées d'un individu, soit que certains liens soient faux, soit par l'absence de liens importants entre différents concepts d'ordre général dit «de superordination $»^{36}$. Comparée à d'autres outils d'évaluation, la carte conceptuelle fait apparaître le caractère contextuel de l'erreur ainsi que les structures explicatives sous-jacentes, correspondant le plus souvent à une logique qui peut faire obstacleà la construction du savoir. On rappellera que selon l'approche cognitiviste, l'apprentissage ne résulte pas uniquement de l'ajout de connaissances nouvelles à la structure de connaissances préalable, mais qu'il peut également consister en de nouvelles mises en liens de connaissances préexistantes ${ }^{37}$. L'utilisation des cartes conceptuelles comme outils d'évaluation sommative (sanctionnelle) a fait l'objet de nombreuses études, y compris dans le domaine médical. Ces études ont tenté de démontrer les qualités docimologiques de I'outili, 38. Tout d'abord, I'introduction d'une nouvelle modal ité évaluative nécessite que les apprenants aient été formés à son utilisation. En effet, une maîtrise insuffisante de la technique d'élaboration d'une carte conceptuelle aura une influence sur la production de l'étudiant et donc sur la fidélité et la validité de l'outi ${ }^{39}$. La pertinence et la validité de contenu des cartes conceptuelles pour éval uer l'apprentissage des étudiants, et plus spécifiquement la façon dont ils organisent leurs connaissances, sont rarement mises en cause. II est, en général, démontré qu'une bonne organisation des connaissances est prédictive de l'expertise et que peu d'outils d'évaluation permettent d'apprécier la façon dont un apprenant construit et organise ses connaissances. Cependant, s'il est démontré que la carte conceptuelle révèle les évolutions en termes d'organisation et de qualité des connaissances, les résultats obtenus ne sont pas toujours corrélés aux autres tests (les études ${ }^{37}$ montrent de grandes variations de corrélation, allant du quasi zéro à des corrélations situées entre 0.50 et 0.80 ). Selon certains auteurs, dans le domaine médical, la validité et la fidélité de l'instrument nécessitent d'être démontrées par de nouvel les étude ${ }^{6,40}$. D e surcroît, la pondération des cartes conceptuelles (c'est-à-dire l'attribution d'une valeur numérique aux différents éléments constitutifs de la carte dans le but d'élaborer un score) reste souvent difficile et variable. Elle dépend de l'expé rience des participants à élaborer une carte conceptuelle et des modalités d'élaboration elles-mêmes ${ }^{39,41,42}$. C es différentes variables auraient une influence sur la validité prédictive des cartes conceptuelles ${ }^{43}$. Enfin, la carte conceptuelle se révèle d'autant plus commode d'utilisation que la méthodologie d'élaboration est précise et directive (compléter une carte, par exemple). A partir du moment où les étudiants ont été formés à élaborer des cartes conceptuelles (temps plus ou moins long en fonction des modal ités d'élaboration), la durée nécessaireà la réal isation d'une carte conceptuelle semble comparable à celle d'un examen classique (environ 30 minutes) ; le temps de correction varierait en fonction de la pondération proposée et du degré de prépara tion des correcteurs (entre 1 et 5 minutes selon certains auteurs) ${ }^{39}$.

Q u'elle soit formative ou sommative, l'analyse de la 


\section{Références}

carte conceptuelle se réfère à des critères. II est possible de pondérer une carte conceptuelle, de la comparer à une carte d'expert, et/ou d'associer ces deux modes $d^{\prime}$ analyse ${ }^{38}$. N ovak et G owin ${ }^{7}$ ont proposé des critères d'analyse basés sur les théories de l'apprentissage issues de la psychologie cognitive, en référence ou non avec une carte d'experts. Analyser une carte conceptuelle en se référant à celle d'un ou plusieurs experts repose sur le principe qu'il existe une représentation idéale pour un thème donné. La validité d'une telle conception norma- tive de l'organisation des connaissances est cependant discutable. On sait en effet que, tant les processus de résolution de problèmes que les représentations cognitives sur lesquels ces derniers s'appuient sont en grande partie idiosyncrasiques, c'est-à-dire qu'ils sont spécifiques et singuliers à chaque expert ${ }^{44}$. Pour certains auteurs ${ }^{7}$, la comparaison avec la carte d'un expert empêche l'enseignant de prendre en compte l'ensemble des connaissances exprimées par les étudiants.

D 'autres ${ }^{37,43}$ posent la question de la validité de la carte

Tableau 2 : C ritères d'analyse qualitative et quantitative d'une carte conceptuelles, $7,41,44$

\begin{tabular}{|c|c|c|}
\hline Eléments d'analyse & Critères proposés & Pondérations proposées \\
\hline $\begin{array}{l}\text { Qualité des } \\
\text { propositions énoncées } \\
\text { (deux concepts reliés } \\
\text { par un lien explicité) }\end{array}$ & $\begin{array}{l}\text { - Existence deliens } \\
\text { - Validité des liens } \\
\text { - Catégories de liens } \\
\text { - Capacitéde l'apprenant } \\
\text { à illustrer ses propos } \\
\text { àl'aide d'exemples }\end{array}$ & $\begin{array}{l}\text { Points attribués à chaque proposition (deux concepts reliés } \\
\text { par un lien) } \\
\text { Points attribués à chaque catégorie de liens } \\
\text { Points attribués pour chaque concept (caractère plus ou } \\
\text { moins technique du vocabulaire employé) } \\
\text { Points attribués à chaque proposition correcte (en fonction } \\
\text { du degré de précision des liens par exemple) } \\
\text { Points attribués à chaque exemple }\end{array}$ \\
\hline $\begin{array}{l}\text { Qualité de } \\
\text { l'organisation des } \\
\text { connaissances }\end{array}$ & $\begin{array}{l}\text { - Présence de concepts } \\
\text { de superordination } \\
\text { et de concepts } \\
\text { de subordination } \\
\text { - Existence deliens } \\
\text { croisés entreles concepts } \\
\text { de superordination }\end{array}$ & $\begin{array}{l}\text { Points attribués par niveaux hiérarchiques. } \\
\text { Points attribués par liens entre concepts de superordination } \\
\text { (ou domaine de connaissances) }\end{array}$ \\
\hline $\begin{array}{l}\text { Degréde complexité } \\
\text { de la structure } \\
\text { cognitive }\end{array}$ & $\begin{array}{l}\text { - O rganisation en roue ou } \\
\text { étoile: intégration facile de } \\
\text { nouveaux concepts, mais une } \\
\text { seule possibilitéd'accès aux } \\
\text { connaissances (concept } \\
\text { central) } \\
\text { - Organisation en chaîne, } \\
\text { linéaire: rendant difficile } \\
\text { l'intégration de nouveaux } \\
\text { concepts } \\
\text { - O rganisation en réseau : } \\
\text { intégration facile, accès } \\
\text { multipleà la connaissance, } \\
\text { témoignant d'une } \\
\text { compréhension large et } \\
\text { profonde dela part de } \\
\text { l'apprenant }\end{array}$ & Aucune pondération proposée. \\
\hline
\end{tabular}


de référence. Une étude menée pour apprécier les performances prédictives des étudiants d'un cursus scienti fique à l'aide de cartes conceptuelles analysées en fonction de différentes cartes d'experts, montre que les résultats obtenus par les étudiants diffèrent selon les cartes de référence ${ }^{45}$. $D$ 'autres auteurs ont défini des critères d'analyse qualitative portant sur la structuration générale des connaissances ${ }^{45}$. Ces auteurs ont eu le souci de proposer une grille d'analyse d'utilisation simple, basée sur le principe d'intégration progressive décrit par Ausube ${ }^{35}$. Selon ce principe, I'organisation préal able des connaissances va influencer la capacité de l'étudiant à intégrer de nouvelles connaissances. Trois principaux types d'organisation sont décrits en fonction de leur degré de complexité et de leur facilité à intégrer de nouvelles connaissances. Letableau 2 propose un récapitula tif des principaux critères d'analyse d'une carte conceptuelle que l'on peut retrouver dans différentes études menées dans les domaines de l'éducation scientifique et la formation médicale.

\section{Utilisation des cartes conceptuelles dans le domaine de l'éducation thérapeutique des patients atteints de maladie chronique Cadrethéorique del'éducation thérapeutique}

Le domaine de la pédagogie médicale s'étend aujourd'hui à l'éducation thérapeutique ${ }^{47}$. II s'agit pour les soignants, devenus éducateurs, de mettre en œuvre des programmes d'éducation thérapeutiques'adressant à des patients atteints de maladie chronique, afin de développer chez eux des compétences leur permettant de gérer au mieux leur maladie et leur traitement dans leur vie quotidienne ${ }^{48}$. L'éducation thérapeutique institue une nouvelle relation soignant-patient basée sur la rencontre entre deux mondes souvent très différents: le monde biomédical des soignants, modelés par leur formation, et le monde profane des patients, marqués par leurs expériences de la maladie chronique.D ans le domaine de l'éducation thérapeutique, les patients adoptent la position d'apprenant. Pour permettre cette rencontre et favoriser leur apprentissage, il semble essentiel de prendre le temps de mettre en évidence leurs connaissances préalables, mais aussi leurs représentations et leurs croyances. C'est pourquoi la première étape de la démarche d'éducation thérapeutique consiste à réal iser un diagnostic éducatif permettant d'identifier, entre autres éléments, ce que sait déjà le patient sur sa maladie, mais aussi cequ'il a besoin d'apprendre pour mener à bien son ou ses projets de vi $e^{48}$. II est ainsi apparu pertinent de transposer la technique des cartes conceptuelles au domaine de l'éducation thérapeutique, ce qui a fait l'objet d'une importante activité de recherche.

\section{Modalités d'utilisation de la carte conceptuelle en éducation thérapeutique}

D ans un premier temps, nous avons suggéré d'utiliser la technique des cartes conceptuelles sel on une modalité proposée par N ovak et G owin? sous la dénomination de « concept propositional analysis». Elle consiste à produire une carte conceptuelle à partir du discours de l'apprenant, sans structuration préalable. $D$ ans le cadre de I'éducation thérapeutique, cette modalité d'élaboration a l'avantage de ne pas nécessiter d'apprentissage de la part des patients, puisque c'est le soignant qui élabore la carte. D e surcroît, elle représente, selon nous, un support de communication dans la relation soignant-soigné. En revanche, la technique d'entretien, favorisant l'explicitation par le patient de ses connaissances, doit être maîtrisée par les soignants de même que les principes d'élaboration et d'analyse d'une carte conceptuelle. D epuis, d'autres modalités d'utilisation ont été testées : I'élaboration d'une carte conceptuelle par le patient lui-même, avec ou sans phase d'explicitation de ses connaissances, ou par un groupe de patients. Le tableau 3 présente les avantages et les inconvénients des différentes modalités d'élaboration des cartes conceptuelles utilisées en éducation thérapeutique.

\section{Etat des recherches sur les cartes conceptuelles en éducation thérapeutique}

En éducation thérapeutique, les cartes conceptuelles ont principalement été utilisées sur un mode individuel, avec des intentions évaluatives diagnostique et formative. U ne première étude menée auprès de patients diabétiques adultes a permis d'identifier les connaissances antérieures de ces patients, d'apprécier les apprentissages réal isés juste après une éducation thérapeutique et de visualiser l'évolution de ces connaissances et leur appropriation par les patients à distance de l'éducation ${ }^{49}$. Cette étude a égal ement permis d'élaborer une grille d'analyse des cartes conceptuelles basée en partie sur les critères proposés par Gowin et N ovak ${ }^{7}$ et adaptée au cadre de l'éducation thérapeutique (Tableau 4). 


\section{Références}

\section{Tableau 3 : Avantages et inconvénients des différentes modalités d'élaboration des cartes conceptuelles dans le domaine de l'éducation thérapeutique}

\begin{tabular}{|l|}
\hline \multicolumn{1}{|c|}{$\begin{array}{l}\text { Méthode d'élaboration } \\
\text { des cartes conceptuelles }\end{array}$} \\
\hline $\begin{array}{l}\text { Réalisée par le soignant, à partir d'un concept central, } \\
\text { au cours d'un entretien }\end{array}$
\end{tabular}

au cours d'un entretien

$(+)$ : richesse d'informations

$(+)$ : respecte le langage du patient

$(+)$ : facile à mettre en œuvre

(-) : représentation spatiale influencée par le soignant

$(-)$ : hiérarchisation des concepts peu travaillée avec le patient

$(-)$ : analyse longue

Réalisée par le patient, seul dans un premier temps, à partir d'un concept central, après explication de la technique;

pouvant être complétée lors d'un entretien avec le soignant pour permettre l'explicitation des connaissances

(+) : facile à analyser

$(+)$ : respecte le langage du patient

$(+)$ : favorise et respecte l'organisation des concepts par le patient

(-) : nécessite un apprentissage préalable de la part du patient

(-) : moins riche en informations si absence d'explicitation après la phase d'élaboration individuelle

Réal isée par un groupe de patients, à partir d'un concept central, après explication de la technique

$(+)$ : apprentissage possible lors de l'élaboration en groupe

$(+)$ : provoque des conflits cognitifs sources d'apprentissage

$(+)$ : respecte le vocabulaire des patients

$(+)$ : relativement facile à anal yser

$(+)$ : I'accent est mis sur l'organisation des connaissances, la mise en lien des différents concepts

(-) : représentation d'un groupe et non d'un individu

(-) : problèmes posés par les phénomènes de dynamique de groupe

(-) : réal isation longue

Légendes: lessignes + et - indiquent sil sagit plutôt d'un avantage (+) ou d'un inconvénient (-).

Les cartes conceptuelles obtenues au cours d'un entretien d'une trentaine de minutes ont fait apparaître de très larges réseaux de connaissances. La représentation obtenue s'apparente davantage aux réseaux sémantiques sous-tendus par les théories de l'association et dans lesquels les concepts sont reliés entre eux par un ou plusieurs liens de sen $5^{38}$. La figure 2 présente une carte conceptuelle obtenue au cours d'un entretien avec un patient diabétique. $D$ ans ce type de carte conceptuelle, la hiérarchisation des concepts n'apparait pas immédiatement. Ce n'est qu'au cours de l'analyse qu'il est possible d'identifier un système d'organisation des connais sances, avec des domaines de connaissances plus ou moins développés en fonction des préoccupations des 


\begin{tabular}{|c|c|}
\hline \multicolumn{2}{|c|}{$\begin{array}{c}\text { Tableau } 4 \text { : Critères d'analyse d'une carte conceptuelle, utilisés dans le domaine } \\
\text { de l'éducation thérapeutique }{ }^{48,51,52}\end{array}$} \\
\hline $\begin{array}{l}\text { Critères d'analyse } \\
\text { de la nature } \\
\text { de connaissances }\end{array}$ & $\begin{array}{l}\text { Type de connaissances exprimées: déclaratives, procédurales, conditionnelles, } \\
\text { appropriées ou personnalisées (utilisation du «je»). } \\
\text {. N ature des liens exprimés : liens exprimant des généralités, liens de causes à } \\
\text { effets, liens exprimant des conduites à tenir thérapeutiques et préventives, } \\
\text { liens exprimant des opinions personnelles, des affects. } \\
\text {. Validité des connaissances exprimées. } \\
\text { Q Q ualité du vocabulaire employé (degré de technicité). } \\
\text {. Q ual ité des domai nes de connaissances, } \\
\text {. Exhaustivité des connaissances exprimées (connaissances plus ou moins } \\
\text { partielles) }\end{array}$ \\
\hline $\begin{array}{l}\text { Critères d'analyse } \\
\text { de l'organisation } \\
\text { des connaissances }\end{array}$ & $\begin{array}{l}\text { Q ualité des premiers concepts exprimés } \\
\text {. Nombre et qualité des domaines de connaissances (en lien ou pas avec un } \\
\text { concept de superordination) } \\
\text {. N ombre de liens entre les domaines de connaissances } \\
\text {. D omaines de connaissances les plus développés }\end{array}$ \\
\hline
\end{tabular}

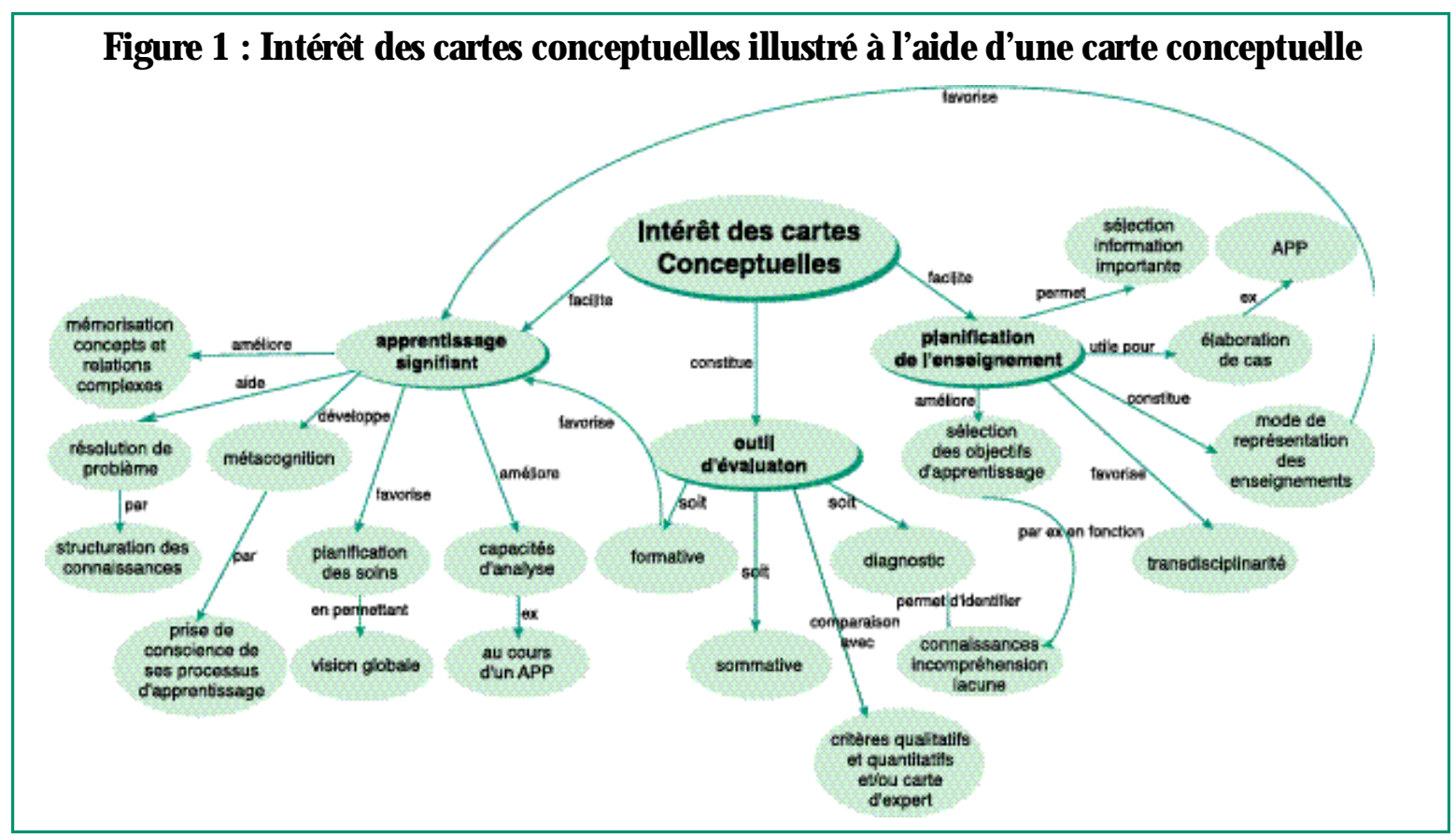

patients au moment de l'élaboration de la carte. Cette première étude a pu montrer à quel point les connaissances préalables étaient nombreuses et résistantes à l'éducation (des connaissances erronées ayant été identifiés avant l'éducation, corrigées par les patients juste après l'éducation et réapparues dans les cartes conceptuelles à distance de l'éducation). D e même, cette recherche a confirmé l'intérêt des cartes conceptuelles pour l'éducation thérapeutique permettant de répondre aux besoins d'apprentissage propres à chaque patient et de mieux comprendre comment les connaissances, les croyances et les émotions s'entremêlent et interagissent, limitant ou favorisant l'apprentissage des patients. 


\section{Références}

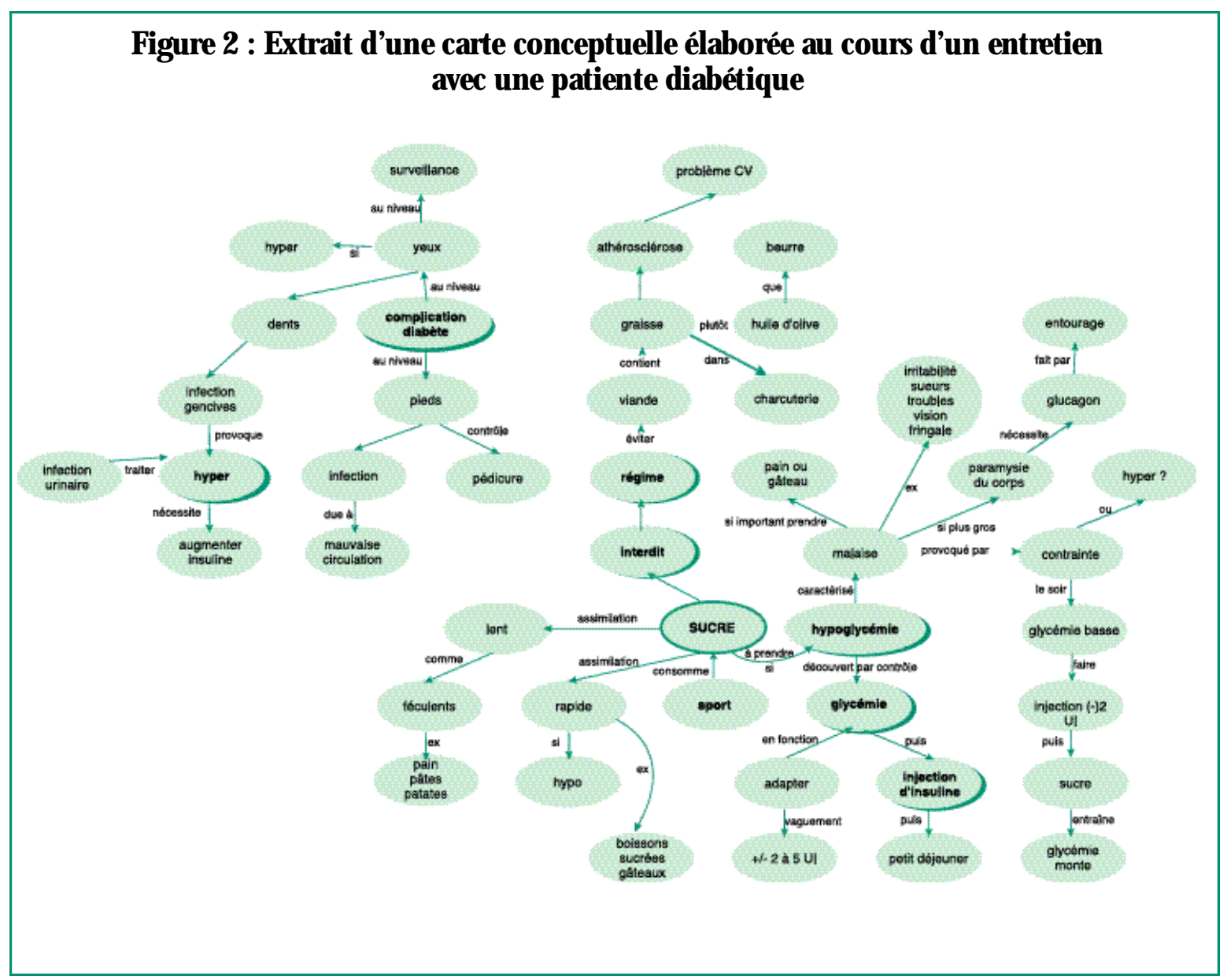

Le même type d'étude a été menée auprès d'enfants diabé tiques âgés de 8 à 13 ans, démontrant la faisabilité et l'inté rêt à utiliser des cartes conceptuelles avec ce public particulier ${ }^{50,51}$. D e mêmes, les cartes conceptuelles ont été employées pour évaluer un programme d'éducation nutritionnelle auprès de patients en surpoids. Les auteurs ont, par ailleurs, mis en lien les informations obtenues dans les cartes conceptuelles avec les résultats de tests psychologiques administrés aux patients ${ }^{52}$. Une autre recherche mené auprès de patients obèses a confirmé l'intérêt diagnostique des cartes conceptuelles en cherchant à repérer dans les cartes conceptuelles des caractéristiques cognitives propres aux patients « normo-évaluateurs » et aux patients «sous-évaluateurs » de leurs apports al imentaires ${ }^{53}$. $D$ 'autres études sont actuellement en cours au sein du Laboratoire de Pédagogie de la Santé de I'U niversité Paris 13 , dont l'un des axes de recherche porte sur l'éducation thérapeutique.

\section{Conclusion}

Les nombreuses recherches, tant dans les domaines de l'éducation et des formations scientifiques, médicales et paramédicales que dans le domaine particulier de l'éducation thérapeutique, démontrent l'intérêt de la technique des cartes conceptuelles. Comme support d'enseignement et d'évaluation formative, elles sont utiles pour aider les personnes à apprendre de façon signifiante et approfondie (meaningful and deep learning). Elles peuvent servir aux formateurs et aux enseignants pour planifier et structurer leurs enseignements ai nsi que pour favoriser la mise en lien entre différentes disciplines. Enfin, utilisées comme moyen d'évaluation, elles semblent être un des rares outils à pouvoir rendre compte des processus d'apprentissagemis en œuvre par les apprenants, ainsi que de leur organisation des connaissances. Cependant, certaines caractéristiques docimologiques de cette technique sont encore aujourd'hui à démonter. 


\section{Références}

1. Wandersee $\mathrm{JH}$. Concept mapping and the cartography of cognition. J Reas Sci Teach $1990 ; 27$ : 923-936.

2. Johsua $S, D$ upin JJ. Introduction à la didactique des sciences et desmathématiques, Paris: PUF, 1993.

3. Baxter GP, Elder AD , Glaser R. Knowledge based cognition and performance assessment in the science classe ment. Educ Psychol 1996 ; 31 : 133-140.

4. Chi M, Fettovich P, Glaser R. Categorization and repre sentation of physics problems by experts and novices CognitiveScience $1981 ; 5: 121-152$.

5. Lesgold A, Rubinson H, Feltovich P, Glaser R, Klopfer D, Wang $Y$. Expertise in a complex skill : diagnostic $x$-ray pictures. In : Chi M TH, Glaser R, Farr M (Eds). The Nature of exepertises. Hillsdale, New Jersey: Lawrence Erlbaum, 1988 : 311-342.

6. West D, Park J, Pomeroy R, Sandoval J. Concept mapping assesment in medical education : a comparison of two scoring systems. M ed Educ 2002, $36: 820-826$.

7. N ovak JD, G owin DB. Learning how to learn, $N$ ew York : Cambridge U niversty Press, (4 $\mathrm{e}^{\mathrm{edition})} 1989$.

8. Rafferty CD , Fleschner LK. Concept M apping : A viable alternative to objective and essay exams. Reading Research and Instruction $1993 ; 32: 25-34$.

9. BriscoeC, LaM aster SU. M eaningful learning in college biology through concept mapping. Am Biol Teach 1991 ; $53: 214-219$.

10. Lawless C, SmeeP O'Shea T. U sing concept sorting and concept mapping in business and public administration, and in education : an overwiew. Educ Res 1998 ; $40: 219-235$.

11. Pinto AJ, Zeitz HJ. Concept mapping : a strategy for promoting meaningful learning in medical education, M ed Teach $1997 ; 19: 114-120$.

12. Schmid R, Telaro G. Concept mapping as an instructional strategy for high school biologyJ Educ Res 1990 ; $84: 78-85$.

13. Stensvold M S, Wilson JT. The interaction of verbal ability with concept mapping in learning from a che mistry laboratory activity. Science Education 1990 ; $74: 473-480$.

14. Tardif J. Pour un enseignement stratégique, l'apport de la psychologie cognitive M ontréal : Ed. Logiques 1992.

15. Gaines C. Concept mapping and synthesizers; instructional strategiesfor encoding and recalling. J N ew York State N urse Ass 1996 : 114-118.
16. Daley BJ. Concept maps : linking nursing theory to clinical nursing pratice. J Cont Nurs Educ 1996 ; $27: 17-27$.

17. All AC, $H$ avens RL. Cognitive/ concept mapping : a teaching strategy for nursing. J Adv N urs 1997 ; 25 : 1210-1219.

18. Baugh N, M ellott K. Clinical concept mapping as pre paration for student nurseśdinical experience. J N urs Educ $1998 ; 37: 253-256$.

19. Rufin F. APP et ensegnement en elearning 2004 [0 n line]. Disponible sur: http://www. cadredesante.com

20. Vygotsky L. Penséeet langage. Paris: M essidor, 1985

21. Okebukola PA, Jegede 0 J. Cognitive preference and learning mode as determinants of meaningful learning through concept mapping. Science Education 1988 ; $72: 489-500$.

22. Johnson DW, Johnson RT. Cooperative learning and achi evement. In : S.Sharan (Ed), Cooperative learning : T heory and research, N ew York : Praeger, 1990 : 23-37.

23. Roth W M , Roychoudhury A. The concept map as a tool of the collaborative construction of knowledge: a microanalysis of high school physics students. J Res Sci Teach $1993 ; 30:$ 503-534.

24. Brown DS. High School Biology: A group Approach to Concept M apping. Am Biol Teacher $2003 ; 65$ : 192-97.

25. Roth W M. Science discourse through collaborative concept mapping: new perspectives for the teacher. $1994 ; 16: 437-455$.

26. Université de $G$ enève. [ $0 n$ line]. $D$ isponible sur : http://edumed.unigech/etudes/formats/app/concept_m ap/exemple_map.php

27. Côté DJ, Bêllavance C, Chamberland M, Graillon A, Un programme pour aider les étudiants en médecineà développer leurs stratégi es d'apprentissage. Pédagogie M édicale2004; 5 : 95-102.

28. $M$ archand $C, G$ agnayre R. U tilisation de la carte conceptuelle auprès de formateurs en santé pour l'apprentissage de concepts pédagogi ques. Pédagogie M édicale $2004 ; 5: 13-23$.

29. M oreira M . Concept maps as tools for teaching. J Coll Sci Teach $1979 ; 9: 283-286$.

30. M c Cagg EC, Dansereau DF. A convergent paradigm for examining knowl edge mapping as a learning strategy.J Educ Res 1991, 84 : 317-324. 


\section{Références}

31. Edmonson K. Concept maps and the development of cases for problem-based learning. Acad M ed 1994 ; $69: 108-110$.

32. H offman E, Trott J, N eely KP. C oncept mapping : a tool to bridge the disciplinary devide. Am J 0 bstet Gynecol $2002 ; 187$ (supp) : 41

33. Chastonay P, Papart J, Laporte J, Praplan G, B renner $E$, Walker $F$ et al. U se of concept mapping to define learning objectives in a master of public health programm. Teach Learn M ed $1999 ; 11$. 21-5.

34. Hadji C. L'évaluati on démystifiée, Paris: ESF, Pratiques et enjeux pédagogiques, 1997.

35. Ausubel D. Educational psychology. A cognitive view., New York : Holt, Rinehart and Winston, ( $2^{\mathrm{e}}$ edition) 1978.

36. I guenane J, M archand C, Ivernois (d') JF. Les cartes sémantiques outil de formation, Les politiquessociales, Belgique1999 : 39-59.

37. Ruiz-Primo M A. Shaveson RJ. Problems and issues in the use of concept maps in science assessment. J Res Sci Teach $1996 ; 33: 569-600$.

38. M cClure JR, Sonak B. Suen H K. Concept $M$ ap Assessment of Classroom L earning : Reliability, Validity and Logistical practicability. J Res Sci Teach 1999 ; $36: 475-92$.

39. West D C, Pomeroy JR, Park JK, Gertenberger EA, Sandoval J. Critical thinking in graduatemedical education : A role for concept M apping assessment ?JAM A $2000 ; 284: 1105-10$.

40. N ovak JD. Clarify vith concept maps. Sci Teach 1991 ; 45-49.

41. Ruiz-Primo M A, Schultz SE, Li M, Shavelson RJ. Comparison of the Reliability and Validity of scores from two Concept-M apping Techniques. J Res Sci Teach $2001 ; 38: 260-78$.

42. Liu X. H inchey M. T he internal consistency of a concept mapping scoring and its effect on predication validity. Int J Sci Educ 1996 ; 18 : 921-37.
43. Acton WH, Johnson PJ, Goldsmith TE. Structural knowledge assessment : Comparison of referant structures. J Educ Psychol 1994 ; 86 : 303-11.

44. Patel VL, Groen GJ.T The general and specific nature of medical expertise: a critical look. In : KA Ericsson and $J$ J. Smith (Eds) Toward a general theory of expertise. Prospects and limits. Cambridge : C ambridge University Press, 1991 : 93-125

45. Kinchin IM , Hay DB, Adams A. H ow a qualitative approach to concept map analysis can be used to aid learning by illustrating patterns of conceptual. Educ Res $2000 ; 42: 45-57$.

46. I vernois (d') JF, Albano M G. Q uand les médecins se font pédagogues. Les C ahiers P édagogiques 2001 ; 399: 55-7.

47. W H O. Therapeutic patient education. Continuing education programmes for healthcare providers in the field of prevention of chronic diseases, Copenhagen, 1998.

48. Ivernois (d') JF, Gaganayre R. Apprendre à éduquer le patient. Approche pédagogique, Paris: M aloine, $\left(2^{\mathrm{e}}\right.$ ed), 2004.

49. M archand C, Ivernois (d') JF, A ssal JP, Slama G, $H$ ivon R. An analysis, using concept mapping, of diabetic patient's knowledge, before and after patient education. M ed Teach $2002 ; 24$ : 90-9.

50. $\mathrm{M}$ archand $\mathrm{C}$. Evaluation pédagogiquedansle domaine del'éducation thérapeutique desenfants. Le Journal des professionnels del'Enfance $2004 ; 27: 64-7$.

51. Pinosa C, M archand C, Tubiana-Rufi N, G agnayreR, Albano M G, Ivernois (d)'JF. The use of concept mapping to enlighten the knowledge networks of diabetic children : a pilot study. Diabetes \& M etabolism 2004, accepté pour publication.

52. França S, I vernois (d') JF, M archand C, Haenni C, Ybarra J, GolayA. Evaluation of nutritional education using concept mapping. Pat Educ Couns $2004 ; 52$ : 183-92.

53. Franca $S, M$ archand $C, C$ raplet $C$, Basdevant $A$, Ivernois (d') JF. Application of « concept mapping » in obese subjects: a pilot study in normo and underreporters, Diabetes \& M etabolism $2003 ; 29: 72-78$. 